\title{
SPECTRA OF TUKEY TYPES OF ULTRAFILTERS ON BOOLEAN ALGEBRAS
}

\author{
JENNIFER A. BROWN AND NATASHA DOBRINEN
}

\begin{abstract}
Extending recent investigations on the structure of Tukey types of ultrafilters on $\mathcal{P}(\omega)$ to Boolean algebras in general, we classify the spectra of Tukey types of ultrafilters for several classes of Boolean algebras, including interval algebras, tree algebras, and pseudo-tree algebras.
\end{abstract}

\section{INTRODUCTION}

The structure of Tukey types of ultrafilters on $\omega$ is the subject of much recent and ongoing research. This line of research was reignited, after Isbell's study in [10, by Milovich in [13. One of the interests in this research stems from the connection via Stone duality between Tukey types of ultrafilters on $\omega$ and cofinal types of neighborhood bases on the Čech-Stone compactification $\beta \omega$ of the natural numbers and its Čech-Stone remainder $\beta \omega \backslash \omega$. Recently, the structure of the Tukey types of ultrafilters on $\omega$ has undergone an explosion of activity, starting with [8] of Dobrinen and Todorcevic, and continuing in work of Milovich in [14, Raghavan and Todorcevic in [15, further work of Dobrinen and Todorcevic in 9 , [7, Blass, Dobrinen, and Raghavan in [1, Dobrinen, Mijares, and Trujillo in [6], and most recently Dobrinen in [5]. These lines of study have all been focused on clarifying the structure of Tukey types of ultrafilters on $\omega$. In particular, the aforementioned works provide good understanding of the Tukey structure for a large class of ultrafilters possessing some means of diagonalization, in particular, p-points. The reader is referred to the recent survey article 4 for more background.

There remain important open problems regarding Tukey types of ultrafilters on $\omega$. One of the most prominent open problems is due to Isbell. In [10, Isbell showed that there are ultrafilters on $\omega$ which have the maximum Tukey type, namely $\left([\mathfrak{c}]^{<\omega}, \subseteq\right)$. He then asked whether there are ultrafilters on $\omega$ which do not have the maximum Tukey type. By work in [8], p-points are never Tukey maximum. However, Shelah constructed a model of ZFC in which there are no p-points (see [18), so what remains of Isbell's problem is the following.

Problem 1. Is there a model of ZFC in which all nonprincipal ultrafilters have the maximum Tukey type?

Milovich showed in $[13$ that there is a nonprincipal ultrafilter $\mathcal{U}$ on $\omega$ such that $(\mathcal{U}, \supseteq)$ is not Tukey maximum if and only if there is a nonprincipal ultrafilter $\mathcal{V}$ on $\omega$ such that $\left(\mathcal{V}, \supseteq^{*}\right)$ is not Tukey maximum. Thus, Isbell's problem for ultrafilters

2010 Mathematics Subject Classification. Primary: 06E05, Secondary: 03G05, 06A07, 03E04. Key words and phrases. Boolean algebra, pseudo-tree algebra, Tukey, ultrafilter.

Dobrinen was supported by National Science Foundation Grant DMS-1301665. 
on the Boolean algebra $\mathcal{P}(\omega)$ is equivalent to the problem for ultrafilters on the Boolean algebra $\mathcal{P}(\omega) /$ fin.

In this paper, we expand the investigation of the Tukey structure of ultrafilters to the general class of all Boolean algebras. The main motivation is the analogue of Isbell's problem for Boolean algebras in general:

Problem 2. Characterize the class of Boolean algebras for which all its nonprincipal ultrafilters have the maximum Tukey type. Further, characterize the class of Boolean algebras which have a nonprincipal ultrafilter which is not of maximum Tukey type.

By understanding the characteristics of Boolean algebras for which we can prove in ZFC whether or not it has more than one Tukey type of nonprincipal ultrafilter, we hope to gain new insight and methods for solving Isbell's problem on $\mathcal{P}(\omega)$ and $\mathcal{P}(\omega) /$ fin.

The second motivation for this line of work is to use the structure of Tukey types of ultrafilters as a means of classifying Boolean algebras. Tukey theory has been successfully used to classify partial orders. This is especially important in cases when isomorphism is too strong a notion to gain any meaningful information. The seminal works of [20, [21, and [19] used Tukey reducibility to classify directed, undirected, and analytic partial orders, respectively. In a similar vein, the structure of the Tukey types of ultrafilters on Boolean algebras can be used as a means for a classifying Boolean algebras. Given a Boolean algebra $\mathbb{B}$, it follows from Stone duality that the Tukey type of an ultrafilter $\mathcal{U}$ on $\mathbb{B}$ is the same as the cofinal type of the neighborhood basis of $\mathcal{U}$ in the Stone space of $\mathbb{B}$. Thus, the study of Tukey types of ultrafilters on Boolean algebras is equivalent to the study of cofinal types of neighborhood bases of Boolean spaces, that is, zero-dimensional compact Hausdorff spaces. Those Boolean algebras with similar Tukey spectra have similar topological properties in their Stone spaces, as the collection of all Tukey types of ultrafilters on a Boolean algebra is equal to the collection of all cofinal types of neighborhood bases of points in the Stone space.

Before stating more precise questions and basic facts, we review the relevant definitions. A partial order $(P, \leq)$ is directed if for any two $p, q \in P$, there is an $r \in P$ such that $p \leq r$ and $q \leq r$. An ultrafilter on a Boolean algebra $\mathbb{B}$ is simply a maximal filter on $\mathbb{B}$. Letting $\leq$ denote the natural partial ordering on a Boolean algebra $\mathbb{B}$ (defined by $a \leq b$ if and only if $a \wedge b=a$ ), we point out that for any ultrafilter $\mathcal{U}$ on a Boolean algebra $\mathbb{B},(\mathcal{U}, \geq)$ is a directed partial ordering. Although Tukey reducibility can be defined for transitive relations in general, we shall assume here that all partial orders discussed are directed, since this article concentrates on ultrafilters.

Given $\left(P, \leq_{P}\right)$, a subset $X \subseteq P$ is cofinal in $P$ if for each $p \in P$, there is an $x \in X$ such that $p \leq_{P} x$. A map $f:\left(P, \leq_{P}\right) \rightarrow\left(Q, \leq_{Q}\right)$ is cofinal if for each cofinal $X \subseteq P$, the image $f[X]$ is cofinal in $Q$. A set $X \subseteq P$ is unbounded if there is no upper bound for $X$ in $P$; that is, there is no $p \in P$ such that for each $x \in X, x \leq_{P} p$. A map $g:\left(P, \leq_{P}\right) \rightarrow\left(Q, \leq_{Q}\right)$ is unbounded or Tukey if for each unbounded $X \subseteq P$, the image $g[X]$ is unbounded in $Q$. Schmidt showed in [17] that the existence of a cofinal map from $\left(P, \leq_{P}\right)$ to $\left(Q, \leq_{Q}\right)$ is equivalent to the existence of a Tukey map from $\left(Q, \leq_{Q}\right)$ to $\left(P, \leq_{P}\right)$. We say that $\left(P, \leq_{P}\right)$ is Tukey reducible to $\left(Q, \leq_{Q}\right)$, and write $\left(P, \leq_{P}\right) \leq_{T}\left(Q, \leq_{Q}\right)$, if there is a cofinal map from $\left(Q, \leq_{Q}\right)$ to $\left(P, \leq_{P}\right)([22])$. 
We say that $\left(P, \leq_{P}\right)$ is Tukey equivalent to $\left(Q, \leq_{Q}\right)$, and write $\left(P, \leq_{P}\right) \equiv_{T}\left(Q, \leq_{Q}\right)$, if and only if $\left(P, \leq_{P}\right) \leq_{T}\left(Q, \leq_{Q}\right)$ and $\left(Q, \leq_{Q}\right) \leq_{T}\left(P, \leq_{P}\right)$.

It follows from work of Schmidt in [17] and is implicit in work of Tukey [22] that, for each infinite cardinal $\kappa$, the directed partial ordering $\left([\kappa]^{<\omega}, \subseteq\right)$ is the maximum among all Tukey types of directed partial orderings of cardinality $\kappa$. That is, every directed partial ordering of cardinality $\kappa$ is Tukey reducible to $\left([\kappa]^{<\omega}, \subseteq\right)$. The minimal Tukey type is 1 (the one-element partially ordered set). An ultrafilter partially ordered by $\geq$ has this minimal Tukey type if and only if it is principal. For directed partial orderings, Tukey types and cofinal types coincide. (See 20] for more background.)

An ultrafilter $\mathcal{U}$ on a Boolean algebra $\mathbb{B}$ has the maximum Tukey type if $(\mathcal{U}, \geq) \equiv_{T}$ $\left([|\mathbb{B}|]^{<\omega}, \subseteq\right)$, which is the maximum possible cofinal type for a directed partial ordering of cardinality $|\mathbb{B}|$. Isbell's result in $[10$ that there is an ultrafilter on $\omega$ which has maximum Tukey type led us to ask whether this is true for Boolean algebras in general: Given a Boolean algebra, is there an ultrafilter which has the maximum Tukey type? We will show that this is not the case: there are several classes of Boolean algebras which do not have any ultrafilters with the maximum Tukey type, the uncountable interval algebras being the simplest of these (see Theorem 24). This along with Problem 2led to the following collection of questions which we investigate. Our intuition was that Boolean algebras with strong structure would yield simple Tukey structures.

Question 3. For which Boolean algebras is there an ultrafilter which has the maximum Tukey type?

Question 4. Are there Boolean algebras on which all ultrafilters have the maximum Tukey type?

Question 5. Which Boolean algebras have ultrafilters with non-maximum Tukey type?

Question 6. Can we characterize those Boolean algebras all of whose ultrafilters have Tukey type strictly below the maximum?

We answer these questions for certain classes of Boolean algebras by obtaining the finer results of classifying their Tukey spectra. By the Tukey spectrum of a Boolean algebra $\mathbb{B}$, denoted $\operatorname{Ts}(\mathbb{B})$, we mean the collection of all Tukey types of ultrafilters on $\mathbb{B}$ partially ordered by Tukey reduction. We use the terminology Tukey spectra of a class $\mathcal{C}$ of Boolean algebras to mean the collection $\{\operatorname{Ts}(\mathbb{B}): \mathbb{B} \in \mathcal{C}\}$. The following is the main focus of this paper.

Problem 7. Given a Boolean algebra, find its Tukey spectrum.

We solve Problem 7 for the classes of free Boolean algebras, superatomic Boolean algebras generated by almost disjoint families, interval algebras, tree algebras, and pseudo-tree algebras.

Section 2 concentrates on classes of Boolean algebras which have an ultrafilter with maximum Tukey type. We present conditions guaranteeing the existence of an ultrafilter with the maximum Tukey type, providing some answers to Question 3. In particular, Theorem 11 shows that every infinite Boolean algebra with an independent family of maximum cardinality has an ultrafilter with maximum Tukey type. In Fact 14 we point out that the class of free Boolean algebras answers 
Question 4 positively. It is then natural to ask whether the completion of a free Boolean algebra has Tukey spectrum consisting of only the maximum Tukey type. In Theorem 19 we rule out certain non-maximum Tukey types: no finite product of regular cardinals is in the Tukey spectrum of the completion of a free Boolean algebra on infinitely many generators. In Theorem 22 we find the Tukey spectra of superatomic Boolean algebras generated by almost disjoint families. In particular, the minimum and maximum Tukey types are always attained.

In Section 3, we classify the Tukey spectra of the families of interval algebras, tree algebras, and pseudo-tree algebras. Theorem 24 classifies the Tukey spectrum of any interval algebra as a collection of products of two cardinals which are determined by adjutting initial and coinitial chains in the linear order. It follows that the Tukey spectrum of the interval algebra of any uncountable linear order does not include the maximum Tukey type. Theorem 28 characterizes the Tukey types of ultrafilters in tree algebras as the product of the cofinality of an initial chain in the tree with the weak product of the cardinality of its set of immediate successors. This immediately leads to Theorem 30 which classifies the Tukey spectra of tree algebras. Lemma 34 distills the essential structure of a set of approximate immediate successors of an initial chain in terms of a $\lambda$-fan (see Definition 33). This is used to classify the Tukey spectra of pseudo-tree algebras in Theorem 36 in terms of the product of an initial chain $C$ and the weak product of the cardinals determined by the $\lambda$-fan of approximate immediate successors above $C$.

In addition to finding the Tukey spectra for these classes of Boolean algebras, we show that for certain prescribed sets of partial orders, a Boolean algebra can be constructed which contains those partial orders in its Tukey spectrum. This is done for interval algebras in Fact 25, and for pseudo-tree algebras in Proposition 32. which also takes care of tree algebras.

We conclude the introduction with some notation and basic facts which will be useful throughout the paper. A function $f:\left(P, \leq_{P}\right) \rightarrow\left(Q, \leq_{Q}\right)$ is called monotone if whenever $p \leq_{P} p^{\prime}$ then $f(p) \leq_{Q} f\left(p^{\prime}\right)$.

Fact 8. (1) Monotone maps with cofinal images are cofinal maps.

(2) Isomorphic partial orders have the same Tukey type.

For $\kappa, \mu$ cardinals, the partial order $\leq$ on $\kappa \times \mu$ is defined coordinate-wise: $(\alpha, \beta) \leq$ $\left(\alpha^{\prime}, \beta^{\prime}\right)$ if and only if $\alpha \leq \alpha^{\prime}$ and $\beta \leq \beta^{\prime}$. More generally, we fix the following notation.

Definition 9. Given a collection of cardinals $\left\{\kappa_{i}: i \in I\right\}$ for some index set $I$, $\prod_{i \in I} \kappa_{i}$ denotes the collection of all functions $f: I \rightarrow \bigcup\left\{\kappa_{i}: i \in I\right\}$ such that for each $i \in I, f(i) \in \kappa_{i}$. The partial ordering $\leq$ on $\prod_{i \in I} \kappa_{i}$ is coordinate-wise: For $f, g \in \prod_{i \in I} \kappa_{i}, f \leq g$ if and only if for all $i \in I, f(i) \leq g(i)$.

Fact 10. (1) For any infinite cardinal $\kappa$, every directed partial ordering of cardinality less than or equal to $\kappa$ is Tukey reducible to $\left([\kappa]^{<\omega}, \subseteq\right)$.

(2) Let $\mathcal{U}$ be an ultrafilter on a Boolean algebra $\mathbb{B}$, and let $\kappa=|\mathbb{B}|$. Then $(\mathcal{U}, \geq) \equiv_{T}\left([\kappa]^{<\omega}, \subseteq\right)$ if and only if there is a subset $\mathcal{X} \subseteq \mathcal{U}$ of cardinality $\kappa$ such that for each infinite $\mathcal{Y} \subseteq \mathcal{X}, \mathcal{Y}$ is unbounded in $\mathcal{U}$.

(3) $(\omega, \leq) \equiv_{T}\left([\omega]^{<\omega}, \subseteq\right)$.

(4) For any uncountable cardinal $\kappa,\left([\kappa]^{<\omega}, \subseteq\right)>_{T}(\kappa, \leq)$.

(5) For any infinite cardinal $\kappa$ and any $n<\omega,(\kappa, \leq) \equiv_{T}\left(\prod_{i<n} \kappa, \leq\right)$. 
(6) Let $\mathcal{U}$ be an ultrafilter on a Boolean algebra $\mathbb{B}$, and let $G \subseteq \mathbb{B}$ be a filter base for $\mathcal{U}$. Then $(\mathcal{U}, \supseteq) \equiv_{T}(G, \supseteq)$.

Proof. (1) follows from work of Schmidt in [17. The proof of (2) is very similar to the proof of Fact 12 in [8. (3) is due to Day in [3]. (4) follows from (1) along with the fact that every countably infinite subset of an uncountable cardinal $\kappa$ is bounded in $\kappa$. To show (5), let $\kappa \geq \omega$ and $n<\omega$. Define $f:(\kappa, \leq) \rightarrow\left(\prod_{i<n} \kappa, \leq\right)$ by $f(\alpha)=\langle\alpha, \ldots, \alpha\rangle$ (the element of $\left(\prod_{i<n} \kappa, \leq\right)$ constant at $\alpha$ ). Then $f$ is an unbounded cofinal map. For (6), note that a filter base $G$ for $\mathcal{U}$ is a cofinal subset of $\mathcal{U}$. Then $(6)$ follows by Fact 3 in 8 .

\section{Boolean algebras attaining the maximum Tukey type}

It is well-known that for each infinite cardinal $\kappa$, there is an ultrafilter on $\mathcal{P}(\kappa)$ with maximum Tukey type. (This follows from combining work of Isbell in [10] and Schmidt in 17.) Such an ultrafilter may be constructed using an independent family on $\kappa$ of cardinality $2^{\kappa}$. We begin by showing that this construction generalizes to any infinite Boolean algebra $\mathbb{B}$ with an independent family of cardinality $|\mathbb{B}|$.

Theorem 11. If $\mathbb{B}$ is an infinite Boolean algebra with an independent family of cardinality $|\mathbb{B}|$, then there is an ultrafilter $\mathcal{U}$ on $\mathbb{B}$ such that $(\mathcal{U}, \geq) \equiv_{T}\left([|\mathbb{B}|]^{<\omega}, \subseteq\right)$.

Proof. Let $\mathbb{B}$ be any infinite Boolean algebra with an independent family $\mathcal{I}=\left\{a_{\alpha}\right.$ : $\alpha<|\mathbb{B}|\}$, and let $\kappa$ denote $|\mathbb{B}|$. Define $\mathcal{J}=\left\{-b: b \in \mathbb{B}\right.$ and $\left\{\alpha<\kappa: b \leq a_{\alpha}\right\}$ is infinite $\}$. We first show that $\mathcal{I} \cup \mathcal{J}$ has the finite intersection property. Let $m, n<\omega$, $\alpha_{i}(i \leq m)$ be distinct members of $\kappa$, and let $b_{j}(j \leq n)$ be distinct members of $\mathcal{J}$. Since each $b_{j}$ is below $a_{\alpha}$ for infinitely many $\alpha<\kappa$, we may choose distinct $\beta_{j}$ $(j \leq n)$ such that for each $j \leq n, b_{j} \leq a_{\beta_{j}}$, and moreover $\beta_{j} \notin\left\{\alpha_{i}: i \leq m\right\}$. Since $\mathcal{I}$ is independent it follows that $\bigwedge_{i \leq m} a_{\alpha_{i}} \wedge \bigwedge_{j \leq n}\left(-a_{\beta_{j}}\right)>\mathbf{0}$. Since each $b_{j} \geq-a_{\beta_{j}}$, we have that $\bigwedge_{i \leq m} a_{\alpha_{i}} \wedge \bigwedge_{j \leq n} b_{j}>\mathbf{0}$.

Let $\mathcal{F}$ be the filter generated by $\mathcal{I} \cup \mathcal{J}$. $\mathcal{F}$ is a proper filter, since its generating set has the finite intersection property.

Claim. $(\mathcal{F}, \geq) \equiv_{T}\left([\kappa]^{<\omega}, \subseteq\right)$.

Proof. It suffices to show that $(\mathcal{F}, \geq) \geq_{T}\left([\kappa]^{<\omega}, \subseteq\right)$, since $\mathcal{F}$ being a directed partial order of size $\kappa$ implies that $(\mathcal{F}, \geq) \leq_{T}\left([\kappa]^{<\omega}, \subseteq\right)$. Define $f:[\kappa]^{<\omega} \rightarrow \mathcal{F}$ by $f(F)=\bigwedge_{\alpha \in F} a_{\alpha}$, for each $F \in[\kappa]^{<\omega}$. We claim that $f$ is a Tukey map. To see this, let $\mathcal{X} \subseteq[\kappa]^{<\omega}$ be unbounded in $\left([\kappa]^{<\omega}, \subseteq\right)$. Then $\mathcal{X}$ must be infinite. The $f$-image of $\mathcal{X}$ is $\left\{\bigwedge_{\alpha \in F} a_{\alpha}: F \in \mathcal{X}\right\}$. Any lower bound $b$ of $\left\{\bigwedge_{\alpha \in F} a_{\alpha}: F \in \mathcal{X}\right\}$ would have to have the property that $b \leq \bigwedge_{\alpha \in \cup \mathcal{X}} a_{\alpha}$. But $\bigvee_{\alpha \in \cup \mathcal{X}}-a_{\alpha}$ is in $\mathcal{B}$, since $\bigcup \mathcal{X}$ is infinite; so the complement of $\bigwedge_{\alpha \in \cup \mathcal{X}} a_{\alpha}$ is in $\mathcal{F}$. Since $\mathcal{F}$ is a proper filter, $\mathcal{F}$ contains no members below $\bigwedge_{\alpha \in \cup \mathcal{X}} a_{\alpha}$. It follows that the $f$ image of $\mathcal{X}$ is unbounded in $\mathcal{F}$. Therefore $f$ is a Tukey map and the claim holds.

Let $\mathcal{U}$ be any ultrafilter on $\mathbb{B}$ extending $\mathcal{F}$. Then $(\mathcal{F}, \geq) \leq_{T}(\mathcal{U}, \geq)$, since the identity map on $(\mathcal{F}, \geq)$ is a Tukey map. Hence, $(\mathcal{U}, \geq) \equiv_{T}\left([\kappa]^{<\omega}, \subseteq\right)$.

The next theorem follows immediately by an application of the Balcar-Franěk Theorem, which states that every infinite complete Boolean algebra has an independent subset of cardinality that of the algebra (see Theorem 13.6 in [12]). 
Theorem 12. Every infinite complete Boolean algebra has an ultrafilter with maximum Tukey type.

Proof. By the Balcar-Franěk Theorem, every infinite complete Boolean algebra $\mathbb{B}$ has an independent family of cardinality $|\mathbb{B}|$. By Theorem 11 , there is an ultrafilter $\mathcal{U}$ on $\mathbb{B}$ such that $(\mathcal{U}, \geq) \equiv_{T}\left([|\mathbb{B}|]^{<\omega}, \subseteq\right)$.

We mention the following theorem of Shelah giving sufficient conditions for a Boolean algebra to have an independent family of maximal size.

Theorem 13 (Shelah, (Theorem 10.1 in [12)). Assume $\kappa, \lambda$ are regular infinite cardinals such that $\mu^{<\kappa}<\lambda$ for every cardinal $\mu<\lambda$, and that $\mathbb{B}$ is a Boolean algebra satisfying the $\kappa$-chain condition. Then every $X \subseteq \mathbb{B}$ of size $\lambda$ has an independent subset of $Y$ of size $\lambda$.

Thus, if $\mathbb{B}$ has the $\kappa$-chain condition, $|\mathbb{B}|=\lambda$, and for all $\mu<\lambda, \mu^{<\kappa}<\lambda$, then $\mathbb{B}$ contains an independent subset of size $|\mathbb{B}|$. Theorem 11 then implies that $\mathbb{B}$ has an ultrafilter with maximum Tukey type.

The next theorem shows that every ultrafilter on a free Boolean algebra has maximum Tukey type. Thus, for each infinite cardinal $\kappa$, the spectrum of the Tukey types of ultrafilters on $\operatorname{Clop}\left(2^{\kappa}\right)$ is precisely $\left\{\left([\kappa]^{<\omega}, \subseteq\right)\right\}$.

Fact 14. For each infinite cardinal $\kappa$ and each ultrafilter $\mathcal{U}$ on $\operatorname{Clop}\left(2^{\kappa}\right),(\mathcal{U}, \geq) \equiv_{T}$ $\left([\kappa]^{<\omega}, \subseteq\right)$.

Proof. Let $\kappa$ be an infinite cardinal. The basic clopen sets of Clop $\left(2^{\kappa}\right)$ are the sets $c_{s}=\left\{f \in 2^{\kappa}: f \supseteq s\right\}$, where $s$ is any function from a finite subset of $\kappa$ into 2 . Given an $x \in 2^{\kappa}$, define a map $f$ from $[\kappa]^{<\omega}$ into the neighborhood base of $x$ by letting $f(F)=c_{x \uparrow F}$. Then $f$ is a cofinal and unbounded map, so the neighborhood base of $x$ has cofinal type exactly $\left([\kappa]^{<\omega}, \subseteq\right)$. Thus, every point in the Stone space $2^{\kappa}$ has neighborhood base of maximum Tukey type. By Stone duality, this implies that each ultrafilter on $\operatorname{Clop}\left(2^{\kappa}\right)$ is Tukey equivalent to $\left([\kappa]^{<\omega}, \subseteq\right)$.

Remark. The Stone space of each free Boolean algebra is homogeneous; that is, given any infinite cardinal $\kappa$, for any two ultrafilters $\mathcal{U}, \mathcal{V}$ on $\operatorname{Clop}\left(2^{\kappa}\right)$, there is a homeomorphism from $\operatorname{Ult}\left(\operatorname{Clop}\left(2^{\kappa}\right)\right)$ onto itself mapping $\mathcal{U}$ to $\mathcal{V}$ (see Exercise 4, page 139 in [12]). Since a homeomorphism maps any neighborhood base of $\mathcal{U}$ cofinally to any neighborhood base of $\mathcal{U}$, and vice versa, $\mathcal{U}$ is Tukey equivalent to $\mathcal{V}$. In fact, homogeneity of the Stone space of any Boolean algebra implies all its ultrafilters have the same Tukey type. However, this says nothing about what that Tukey type is. We shall see in Section 3 that it is possible to have an interval algebra in which all the ultrafilters have the same Tukey type $\kappa$, which is not the maximum type if $\kappa$ is an uncountable cardinal.

Next, we investigate the Tukey spectra of completions of free algebras. By Theorem 12, the completion of $\operatorname{Clop}\left(2^{\kappa}\right)$, denoted r.o. $\left(\operatorname{Clop}\left(2^{\kappa}\right)\right)$, always has an ultrafilter of the maximum Tukey type $\left(\left[2^{\kappa}\right]^{<\omega}, \subseteq\right)$. In particular, the Cohen algebra r.o. $\left(\operatorname{Clop}\left(2^{\omega}\right)\right)$ has an ultrafilter of type $\left([\mathfrak{c}]^{<\omega}, \subseteq\right)$. This leads us to the following question.

Question 15. Do all the ultrafilters in the completion of a free Boolean algebra have maximum Tukey type? 
In Theorem 19, we will rule out some possible Tukey types below the top for all completions of free algebras. We begin with two propositions in which certain completeness or chain condition hypotheses rule out certain Tukey types of ultrafilters.

Proposition 16. If $\kappa \geq \omega$ and $\mathbb{B}$ is a $\kappa^{+}$-complete atomless Boolean algebra, then $\mathbb{B}$ has no ultrafilters of Tukey type $(\kappa, \leq)$.

Proof. Let $\mathcal{U}$ be an ultrafilter on $\mathbb{B}$ and suppose toward a contradiction that there is a strictly decreasing sequence $\left\langle b_{\alpha}: \alpha<\kappa\right\rangle$ cofinal in, and thus generating, $\mathcal{U}$. Without loss of generality, we may assume that for each limit ordinal $\gamma<\kappa$, $b_{\gamma}=\bigwedge_{\alpha<\gamma} b_{\alpha}$ and that $b_{0}=1$. Since $\left\langle b_{\alpha}: \alpha<\kappa\right\rangle$ generates an ultrafilter, it follows that $\bigwedge_{\alpha<\kappa} b_{\alpha}=\mathbf{0}$. For each $\alpha<\kappa$, define $a_{\alpha}=b_{\alpha} \wedge-b_{\alpha+1}$. Since $\mathbb{B}$ is atomless, there are non-zero $a_{\alpha, 0}, a_{\alpha, 1}$ partitioning $a_{\alpha}$. Let $c_{i}=\bigvee_{\alpha<\kappa} a_{\alpha, i}$, for $i<2$. Then $c_{0} \vee c_{1}=\mathbf{1}$ and $c_{0} \wedge c_{1}=\mathbf{0}$; so exactly one of $c_{0}, c_{1}$ must be in $\mathcal{U}$. But for each $i<2$, we have that $c_{i} \geq b_{\alpha}$ for all $\alpha<\kappa$. Since $\left\langle b_{\alpha}: \alpha<\kappa\right\rangle$ generates $\mathcal{U}$, this implies that neither of $c_{0}, c_{1}$ is in $\mathcal{U}$, contradiction.

Proposition 17. Let $\kappa$ be a regular uncountable cardinal. If $\mathbb{B}$ is $\kappa$-c.c., then for all $\lambda \geq \kappa, \mathbb{B}$ has no ultrafilters of Tukey type $(\lambda, \leq)$.

Proof. $\mathbb{B}$ is $\kappa$-c.c. implies there are no strictly decreasing chains of order type $\lambda$ in $\mathbb{B}$ for any $\lambda \geq \kappa$. In particular, no ultrafilter in $\mathbb{B}$ can be generated by a strictly decreasing chain of order type $\lambda$.

The following fact is due to Isbell. Recall that a partial ordering $\left(Q, \leq_{Q}\right)$ is relatively complete if every subset of $Q$ which is bounded from below has a greatest lower bound in $Q$.

Proposition 18 (Isbell, 10]). Let $\left(P, \leq_{P}\right)$ and $\left(Q, \leq_{Q}\right)$ be directed partial orderings. If $Q$ is relatively complete, then $P \geq_{T} Q$ if and only if there exists a monotone map $f: P \rightarrow Q$ which has cofinal range.

Theorem 19. Let $\kappa$ be an infinite cardinal. Then each ultrafilter on the completion of the free algebra on $\kappa$ generators is not Tukey reducible to $\prod_{i \leq n} \kappa_{i}$ for any finite collection of regular cardinals $\left\{\kappa_{i}: i \leq n\right\}$.

Proof. Let $\kappa$ be an infinite cardinal and $\mathcal{U}$ be any ultrafilter on r. o. $\left(\operatorname{Clop}\left(2^{\kappa}\right)\right)$. We will show that for any collection of finitely many regular cardinals $\kappa_{i}, i \leq n$, $\prod_{i \leq n} \kappa_{i} \Varangle_{T} \mathcal{U}$. It suffices to consider only infinite cardinals $\kappa_{i}$, as all ultrafilters on r. o. $\left(\operatorname{Clop}\left(2^{\kappa}\right)\right)$ are nonprincipal since r. o. $\left(\operatorname{Clop}\left(2^{\kappa}\right)\right)$ is atomless. Since $\mathcal{U}$ is an ultrafilter, it is upwards closed. In particular, the directed partial ordering $(\mathcal{U}, \geq)$ is relatively complete. Thus, by Proposition[18, whenever $\left(P, \leq_{P}\right) \geq_{T}(\mathcal{U}, \geq)$, there is a monotone cofinal map witnessing this.

Suppose $n=0$ and let $\kappa_{0}$ be any regular infinite cardinal. If $\kappa_{0} \geq_{T} \mathcal{U}$, then by Proposition 18, there is a monotone cofinal map from $\kappa_{0}$ into $\mathcal{U}$. Any monotone map will take $\kappa_{0}$ to a decreasing sequence in $\mathcal{U}$, which is either eventually constant or else has a strictly decreasing subsequence of cofinality $\kappa_{0}$. Propositions [16] and 17 imply that $\mathcal{U}$ is not generated by any strictly decreasing infinite sequence of members of $\mathcal{U}$. Since $\mathcal{U}$ is not principal it cannot be generated by a single member. Thus, $\kappa_{0} \Varangle_{T} \mathcal{U}$.

Now suppose that $n \geq 1$ and given any infinite regular cardinals $\kappa_{i}, i<n$, $\prod_{i<n} \kappa_{i} \Varangle_{T} \mathcal{U}$. Let $\left\{\kappa_{i}: i \leq n\right\}$ be any collection of infinite regular cardinals, 
and without loss of generality, assume they are indexed in strictly increasing order. Suppose that $f: \prod_{i \leq n} \kappa_{i} \rightarrow \mathcal{U}$ is a monotone map into $\mathcal{U}$. Fix a sequence $\bar{\alpha} \in$ $\prod_{i<n} \kappa_{i} . f$ maps the strictly increasing sequence $\left\langle\bar{\alpha}-\beta: \beta \in \kappa_{n}\right\rangle$ in $\prod_{i \leq n} \kappa_{i}$ to a decreasing (not necessarily strictly decreasing) sequence in $\mathcal{U}$, since $f$ is monotone. Since $\kappa_{n}$ is uncountable and $\mathcal{U}$ has no uncountable strictly decreasing sequences, there is some $\beta(\bar{\alpha}) \in \kappa_{n}$ such that $f(\bar{\alpha} \frown \beta(\bar{\alpha}))=\min \left\{f(\bar{\alpha} \frown \beta): \beta \in \kappa_{n}\right\}$. Let $\gamma$ denote $\sup \left\{\beta(\bar{\alpha}): \bar{\alpha} \in \prod_{i<n} \kappa_{i}\right\}$. Since $\left|\prod_{i<n} \kappa_{i}\right|=\kappa_{n-1}<\kappa_{n}$, it follows that $\gamma<\kappa_{n}$. Since $f$ is monotone, the $f$-image of $\prod_{i \leq n} \kappa_{i}$ is bounded below in $\mathcal{U}$ by the set $\left\{f\left(\bar{\alpha}^{\frown} \gamma\right): \bar{\alpha} \in \prod_{i<n} \kappa_{i}\right\}$. By the induction hypothesis, this is not possible, since the set $\left\{(\bar{\alpha}) \frown \gamma: \bar{\alpha} \in \prod_{i<n} \kappa_{i}\right\}$ is isomorphic as a partially ordered set to $\prod_{i<n} \kappa_{i}$. Thus, $f$ cannot be a cofinal map.

Therefore, there is no monotone cofinal map from $\prod_{i \leq n} \kappa_{i}$ into $\mathcal{U}$. Hence, by Proposition 18, $\prod_{i \leq n} \kappa_{i} \Varangle_{T} \mathcal{U}$.

In particular, the Cohen algebra has no ultrafilters of Tukey type $1, \omega, \omega_{1}, \mathfrak{c}$, $\omega \times \omega_{1}$, or any finite product of regular cardinals $\kappa_{i}, i \leq n$, where $\omega \leq \kappa_{0}<\kappa_{1}<$ $\cdots<\kappa_{n} \leq \mathfrak{c}$.

Question 20. Does r. o. $\left(\operatorname{Clop}\left(2^{\kappa}\right)\right)$ have an ultrafilter Tukey equivalent to $\prod_{i \in I} \kappa_{i}$ for some infinite collection of regular infinite cardinals? In particular, does the Cohen algebra have an ultrafilter Tukey equivalent to $\left(\omega^{\omega}, \leq\right)$ ?

Remark. Milovich in 14] defines a preorder to be cofinally rectangular if it is cofinally equivalent to the product of some finite collection of regular cardinals. Theorem 19 shows that all ultrafilters on the completion of a free Boolean algebra (on infinitely many generators) are not cofinally rectangular. We will show in Section 3. Theorem 24, that there are Boolean algebras (namely interval algebras) which have only cofinally rectangular ultrafilters.

The following simple fact shows that each finite-cofinite Boolean algebra has Tukey spectrum of size two, consisting exactly of the minimum and the maximum Tukey types.

Fact 21. Let $X$ be any infinite set, and let $\mathbb{B}$ denote the finite-cofinite algebra on $X$. Then the ultrafilters on $\mathbb{B}$ consist exactly of the principal ultrafilters and the cofinite ultrafilter. Thus, the Tukey spectrum of $\mathbb{B} i s\left\{(1, \leq),\left([|X|]^{<\omega}, \subseteq\right)\right\}$.

Proof. The ultrafilter of cofinite subsets of $X$ is isomorphic to $[|X|]^{<\omega}$. If an ultrafilter on $\mathbb{B}$ contains a finite set, then it is a principal ultrafilter.

This section closes with the Tukey spectra of Boolean algebras generated by some almost disjoint family on an infinite set. Let $\lambda$ be an infinite cardinal. A family $\mathcal{A} \subseteq \mathcal{P}(\lambda)$ is almost disjoint if for all pairs $a, b \in \mathcal{A},|a \cap b|<\omega$. Given an almost disjoint family $\mathcal{A} \subseteq \mathcal{P}(\lambda)$, the almost disjoint Boolean algebra generated by $\mathcal{A}$ is the subalgebra of $\mathcal{P}(\lambda)$ generated by $\mathcal{A} \cup[\lambda]^{<\omega}$. Any Boolean algebra generated from an almost disjoint family is superatomic. (See Example 0.1 on page 721 in [16.)

Theorem 22. Let $\mathcal{A}$ be an almost disjoint family on an infinite cardinal $\lambda$ with $|\mathcal{A}|=\kappa$, and let $\mathbb{B}$ denote the subalgebra of $\mathcal{P}(\lambda)$ generated by $\mathcal{A}$. Then every ultrafilter on $\mathbb{B}$ has Tukey type 1 or $[\mu]^{<\omega}$ for some $\omega \leq \mu \leq \kappa$. The minimum and maximum types, 1 and $[\kappa]^{<\omega}$, are always realized. For $\omega \leq \mu<\kappa,[\mu]^{<\omega}$ is realized as the Tukey type of some ultrafilter on $\mathbb{B}$ if and only if there is an a $\in \mathcal{A}$ such that the set $\{a \cap-b: b \in \mathcal{A} \backslash\{a\}\}$ has cardinality $\mu$. 
Proof. Let $\mathcal{A}$ be an almost disjoint family on an infinite cardinal $\lambda$. Let $\mathbb{B}$ denote the subalgebra of $\mathcal{P}(\lambda)$ generated by $\mathcal{A}$, and let $\kappa=|\mathbb{B}|$. The ultrafilters on $\mathbb{B}$ are of three possible forms, each of which is realized: principal, generated by $\{-a: a \in \mathcal{A}\}$, or generated by $\{a \cap-b: b \in \mathcal{A} \backslash\{a\}\}$ for some $a \in \mathcal{A}$. The principal ultrafilters have Tukey type 1.

Suppose $\mathcal{U}$ is the ultrafilter generated by the set $\{-a: a \in \mathcal{A}\}$. In this case, index the members of $\mathcal{A}$ so that $\mathcal{A}=\left\{a_{\alpha}: \alpha<\kappa\right\}$. This ultrafilter is nonprincipal. As above, the map $g:[\kappa]^{<\omega} \rightarrow \mathcal{U}$, given by $g(F)=\bigcap_{\alpha \in F}-a_{\alpha}$, is a monotone cofinal Tukey map. Therefore, $(\mathcal{U}, \geq) \equiv_{T}\left([\kappa]^{<\omega}, \subseteq\right)$, since $|\mathbb{B}|=\kappa$.

For the third type of ultrafilter, fix any $a \in \mathcal{A}$ and enumerate $\mathcal{A} \backslash\{a\}$ as $\left\{a_{\alpha}\right.$ : $\alpha<\kappa\}$, and let $\mathcal{U}$ be the ultrafilter generated by $\{a\} \cup\left\{-a_{\alpha}: \alpha<\kappa\right\}$. Let $K \subseteq \kappa$ be a maximal subset of $\kappa$ such that for all $\alpha \neq \beta$ in $K, a \cap-a_{\alpha} \neq a \cap-a_{\beta}$, and let $\mu=|K|$. Without loss of generality, suppose $\mathcal{U}$ is nonprincipal, and hence $\mu \geq \omega$. Define a map $g:[K]^{<\omega} \rightarrow \mathcal{U}$ by $g(F)=a \cap\left(\bigcap_{\alpha \in F}-a_{\alpha}\right)$, for each $F \in[K]^{<\omega}$. We claim that $g$ is a monotone cofinal and Tukey map. By definition, it is clear that $g$ is monotone. Since its range is all of $\mathcal{U}$, it is a cofinal map. To check that $g$ is Tukey, let $\mathcal{X} \subseteq[K]^{<\omega}$ and suppose that there is a bound $b \in \mathcal{U}$ for $\{g(F): F \in \mathcal{X}\}$. Then $b \subseteq a \cap\left(\bigcap_{\alpha \in F}-a_{\alpha}\right)$, for each $F \in \mathcal{X}$. Letting $G=\bigcup \mathcal{X}$, we see that $b \subseteq a \cap\left(\bigcap_{\alpha \in G}-a_{\alpha}\right)$. Thus, $G$ must be finite; hence also $\mathcal{X}$ is finite and therefore bounded. Therefore, $\mathcal{U} \equiv_{T}[\mu]^{<\omega}$.

Question 23. What are the Tukey spectra of superatomic Boolean algebras in general?

Lastly, we state a theorem that will be proved in Section 4: A tree algebra Treealg $T$ of size $\kappa$ has an ultrafilter with maximum Tukey type if and only if the underlying tree $T$ has an initial chain with $\kappa$-many immediate successors. (This is Corollary 29, we will also prove in Corollary 36 a more general version for pseudotree algebras.)

\section{Spectra of Tukey types of interval algebras, tree algebras, and PSEUDO-TREE ALGEBRAS}

Basic facts about interval algebras and tree algebras can be found in Volume 1 of the Handbook of Boolean Algebras [12; basic facts about pseudo-tree algebras which generalize from corresponding tree algebra facts can be found in [11. Since pseudo-trees are probably the least well-known of these classes, we provide some background on them here. We follow the notation in [11].

A pseudo-tree is a partially ordered set $(T, \leq)$ such that for each $t \in T$, the set $T \downarrow t=\{s \in T: s \leq t\}$ is linearly ordered. The pseudo-tree algebra on a pseudo-tree $T$ is generated in the same way as a tree algebra: $\operatorname{Treealg}(T)$ is the algebra of sets generated by the "cones" $T \uparrow t=\{s \in T: s \geq t\}$, for $t \in T$. A pseudo-tree algebra is thus a generalization of both an interval algebra and a tree algebra, and the following discussion of the correspondence between ultrafilters and initial chains applies to all three classes of Boolean algebras.

Let $T$ be an infinite pseudo-tree with a single root. (It does no harm to assume that all of our pseudo-trees have single roots; any pseudo-tree algebra is isomorphic to a pseudo-tree algebra on a pseudo-tree with a single root (see [1]).) An initial chain in $T$ is a non-empty chain $C \subseteq T$ such that if $c \in C$ and $t<c$ then $t \in C$. There is a one-to-one correspondence between ultrafilters $\mathcal{U}$ on Treealg $T$ and initial 
chains in $T$, given by

$$
\phi(\mathcal{U})=\{t \in T: T \uparrow t \in \mathcal{U}\} .
$$

The inverse of this map shows how ultrafilters are generated by sets defined in terms of their corresponding initial chains: for $C$ an initial chain in $T, \phi^{-1}(C)=\left\langle H_{C}\right\rangle$, where

$$
H_{C}=\left\{(T \uparrow t) \backslash \bigcup_{s \in S}(T \uparrow s): t \in C, S \text { is a finite antichain of elements } s>C\right\}
$$

(where $S$ is allowed to be empty). This set of generators is closed under finite intersection, and $(\mathcal{U}, \supseteq) \equiv_{T}\left(H_{C}, \supseteq\right)$.

Let $C \subseteq T$ be an initial chain. Call a subset $R \subseteq T$ a set of approximate immediate successors of $C$ if

(i) $r>C$ for all $r \in R$, and

(ii) for all $s>C$, there is an $r \in R$ such that $C<r \leq s$. Then define

$$
\varepsilon_{C}=\min \{|R|: R \text { is a set of approximate immediate successors of } C\} .
$$

The character $\chi$ of an ultrafilter $\mathcal{U}$ on Treealg $T$ is the minimum size of a generating set for $\mathcal{U}$. If $C$ is the initial chain corresponding to $\mathcal{U}$, then $\chi(\mathcal{U})=$ $\max \left\{\varepsilon_{C}, \operatorname{cf} C\right\}$ (see [2]). If $T$ is a tree, then the set $\operatorname{imm}(C)$ of immediate successors of an initial chain $C$ in $T$ is well-defined, even if $C$ does not have a top element, and $\varepsilon_{C}=|\operatorname{imm}(C)|$.

We describe all possible Tukey types of ultrafilters on interval algebras in Theorem 24. From this, we classify the spectra of Tukey types of all interval algebras. In the terminology of Milovich [14, ultrafilters on interval algebras have cofinally rectangular Tukey types. We assume that all linear orders mentioned have least elements. (Where a linear order does not naturally have a least element, we add an element $-\infty$ to $L$ and proceed to build Intalg $L$ as in [12.)

For $L$ a linear order and $X \subseteq L$, the coinitiality of $X$, denoted $\operatorname{ci}(X)$, is the least cardinal $\mu$ such that $\mu^{*}$ is coinitial in $X$.

Theorem 24. Let $L$ be a linear ordering. Let $P$ denote the set of pairs of regular cardinals $(\kappa, \mu)$ for which there is an initial chain $C$ in $L$ such that the cofinality of $C$ is $\kappa$ and the coinitiality of $L \backslash C$ is $\mu$. Then the Tukey spectrum of Intalg $L$ is exactly $\{(\kappa \times \mu, \leq):(\kappa, \mu) \in P\}$.

Proof. Let $L$ be a linear order with a first element and set $A=\operatorname{Intalg} L$. Since $A$ is also a pseudo-tree algebra, its ultrafilters are associated with initial chains as are those of pseudo-tree algebras. Let $\mathcal{U} \in$ Ult $A$ and let $C$ be the initial chain associated with $\mathcal{U}$. Let $\kappa=\operatorname{cf} C$ and $\left\{c_{\alpha}: \alpha<\kappa\right\}$ be an increasing cofinal sequence in $C$. Let $\mu=\operatorname{ci}(L \backslash C)$ and let $\left\{l_{\beta}: \beta<\mu\right\}$ be a decreasing coinitial sequence in $L \backslash C$. Letting

$$
G=\left\{\left[c_{\alpha}, l_{\beta}\right): \alpha<\kappa, \beta<\mu\right\},
$$

we see that $\mathcal{U}=\langle G\rangle$, and hence $(\mathcal{U}, \supseteq) \equiv_{T}(G, \supseteq)$. Define $f:(G, \supseteq) \rightarrow(\kappa \times \mu)$ by $f\left(\left[c_{\alpha}, l_{\beta}\right)\right)=(\alpha, \beta)$. One can check that $f$ is an unbounded cofinal map, so that $(G, \supseteq) \equiv_{T}(\kappa \times \mu, \leq)$.

Thus if $L$ is a linear order and $\mathcal{U}$ is an ultrafilter on Intalg $L$, then there are only three possibilities for the Tukey type of $(\mathcal{U}, \supseteq)$. Letting $C$ be the initial chain corresponding to $\mathcal{U}$, either 
(1) $(\mathcal{U}, \supseteq) \equiv_{T}(\kappa, \leq)$ where cf $C=\kappa$, or

(2) $(\mathcal{U}, \supseteq) \equiv_{T}(\mu, \leq)$ where $\operatorname{ci}(L \backslash C)=\mu$, or

(3) $(\mathcal{U}, \supseteq) \equiv_{T}(\kappa \times \mu, \leq)$ where $\operatorname{cf} C=\kappa$ and $\operatorname{ci}(L \backslash C)=\mu$.

(Recall from Fact 10 (5) that if $\operatorname{cf} C=\kappa=\operatorname{ci}(L \backslash C)$ then $(\mathcal{U}, \supseteq) \equiv_{T}(\kappa \times \kappa, \leq) \equiv_{T}$ $(\kappa, \leq)$.)

Observe that Fact 10 (4) then implies that no uncountable interval algebra Intalg $L$ has an ultrafilter of maximal Tukey type $\left([|L|]^{<\omega}, \subseteq\right)$. Countably infinite linear orders always have ultrafilters of top Tukey type: let $L$ be a countably infinite linear order. Then $L$ contains a initial chain $C$ with $\operatorname{cf} C=\omega$ or $\operatorname{ci}(L \backslash C)=\omega$; and in any case, if $\mathcal{U}$ is the ultrafilter corresponding to $C,(\mathcal{U}, \supseteq) \equiv_{T}(\omega, \leq) \equiv_{T}\left([\omega]^{<\omega}, \subseteq\right)$.

Next, we show that the Tukey spectra of interval algebras is quite robust.

Fact 25. Given any collection $P$ of pairs of regular cardinals, each of which is either 1 or else is infinite, there is a linear order $L$ whose Tukey spectrum includes $\{(\kappa \times \mu, \leq):(\kappa, \mu) \in P\}$.

Proof. Let $\lambda=|P|$ and enumerate the pairs of regular cardinals in $P$ as $\left\{\left(\kappa_{\alpha}, \mu_{\alpha}\right)\right.$ : $\alpha<\lambda\}$. For $\alpha<\lambda$, let $X_{\alpha}$ be a sequence of order type $\kappa_{\alpha}$, and let $Y_{\alpha}$ be a sequence of order type $\mu_{\alpha}^{*}$. Let $L$ be the linear order

$$
L=\bigcup_{\alpha<\lambda}\{\alpha\} \times\left(X_{\alpha} \frown Y_{\alpha}\right)
$$

with the lexicographic ordering. For $\alpha<\lambda$, let $C_{\alpha}$ denote the set of those elements of $L$ that are below every element of $Y_{\alpha}$. Then $\operatorname{cf} C_{\alpha}=\operatorname{cf} X_{\alpha}=\kappa_{\alpha}$ and $\operatorname{ci}\left(L \backslash C_{\alpha}\right)=$ ci $Y_{\alpha}=\mu_{\alpha}$. Letting $\mathcal{U}_{\alpha}$ be the ultrafilter corresponding to $C_{\alpha}$, it follows that $\left(\mathcal{U}_{\alpha}, \supseteq\right) \equiv_{T}\left(\kappa_{\alpha} \times \mu_{\alpha}, \leq\right)$.

We note that the Tukey spectrum of the interval algebra in the proof of Fact 25 may also contain types not among $\{(\kappa \times \mu, \leq):(\kappa, \mu) \in P\}$. For example, if $P=\left\{\left(\omega_{1}, \omega_{1}\right)\right\}$, then the construction gives us $L=\omega_{1}+\omega_{1}^{*}$, and $\operatorname{Intalg}(L)$ has the type $(\omega, \leq)$ in its Tukey spectrum.

Now we attend to the class of tree algebras. All products we mention in what follows will be of the following weak sort: for an index set $I$ and a collection of cardinals $\left\{\kappa_{i}: i \in I\right\}$, let $\prod_{i \in I}^{\mathrm{w}} \kappa_{i}$ denote the collection of all functions $f \in \prod_{i \in I} \kappa_{i}$ such that for all but finitely many $i \in I, f(i)=0$; again the partial ordering is coordinate-wise. In the special case where the index set $I$ is a cardinal and each $\kappa_{i}$ is 2 , we have the following fact.

Fact 26. For any $\kappa, \prod_{\alpha<\kappa}^{\mathrm{w}}\{0,1\} \equiv_{T}\left([\kappa]^{<\omega}, \subseteq\right)$.

Proof. For $F \in[\kappa]^{<\omega}$, set $f(F)=\left\langle e_{\alpha}: \alpha<\kappa\right\rangle$ where

$$
e_{\alpha}=\left\{\begin{array}{l}
1 \text { if } \alpha \in F \\
0 \text { if } \alpha \notin F
\end{array}\right.
$$

Then $f$ is an unbounded cofinal map.

Proposition 27 and Corollary 29 characterize those trees $T$ for which the algebra Treealg $T$ has an ultrafilter of maximal Tukey type $\left([|T|]^{<\omega}, \subseteq\right)$.

Proposition 27. If $T$ is a tree of size $\omega$ with a single root, then Treealg $T$ has an ultrafilter of type $\left([\omega]^{<\omega}, \subseteq\right)$. 
Proof. Let $T$ be a tree of size $\omega$ with a single root. Then also $|\operatorname{Treealg} T|=\omega$. If there is some $z \in T$ with $\omega$-many immediate successors $\left\{s_{i}: i<\omega\right\}$, then set $C=$ $T \downarrow z$ and let $\mathcal{U}=\phi^{-1}(C)$ be the corresponding ultrafilter. Define $a_{n} \in \operatorname{Treealg} T$, for $n<\omega$, by $a_{n}=(T \uparrow z) \backslash \bigcup_{i<n}\left(T \uparrow s_{i}\right)$. Then the $a_{n}$ form a chain of type $\omega$ in $(\mathcal{U}, \supseteq)$, so that $(\mathcal{U}, \supseteq) \equiv_{T}(\omega, \leq) \equiv_{T}\left([\omega]^{<\omega}, \subseteq\right)$.

Otherwise, no $z \in T$ has $\omega$-many immediate successors. Since $T$ has a single root, this means that for all $n<\omega,\left|\operatorname{Lev}_{n}(T)\right|<\omega$; that is, $T$ is an $\omega$-tree. By König's Lemma, $T$ has an infinite chain $\left\{c_{n}: n<\omega\right\}$. Let $C$ be minimal among initial chains containing $\left\{c_{n}: n<\omega\right\}$. Let $\mathcal{U}=\phi^{-1}(C)$ be the ultrafilter corresponding to $C$. For each $n<\omega$, set $a_{n}=T \uparrow c_{n}$. Then the $a_{n}$ form a chain of type $\omega$ in $(\mathcal{U}, \supseteq)$, so that $(\mathcal{U}, \supseteq) \geq_{T}(\omega, \leq)$. Since $(\omega, \leq)$ has the maximum Tukey type for $\omega$, $(\mathcal{U}, \supseteq) \equiv_{T}\left([\omega]^{<\omega}, \subseteq\right)$.

Theorem 28. Let $T$ be a tree, let $\mathcal{U}$ be an ultrafilter on $T$, and let $C$ be the initial chain corresponding to $\mathcal{U}$. Let $\left\{c_{\alpha}: \alpha<\operatorname{cf} C\right\}$ be an increasing cofinal sequence in $C$, and let $\left\{s_{\beta}: \beta<\mu\right\}$ be the set of immediate successors of $C$ in $T$. Then $(\mathcal{U}, \supseteq) \equiv_{T}\left(\operatorname{cf} C \times \prod_{\beta<\mu}^{\mathrm{w}}\{0,1\}, \leq\right)$.

Proof. $\mathcal{U}$ is generated by $G=\left\{\left(T \uparrow c_{\alpha}\right) \backslash \bigcup_{\beta \in F}\left(T \uparrow s_{\beta}\right): \alpha<\operatorname{cf} C, F \in[\mu]^{<\omega}\right\}$. Define $f:(G, \supseteq) \rightarrow\left(\operatorname{cf} C \times \prod_{\beta<\mu}^{\mathrm{w}}\{0,1\}, \leq\right)$ by $f\left(\left(T \uparrow c_{\alpha}\right) \backslash \bigcup_{\beta \in F}\left(T \uparrow s_{\beta}\right)\right)=$ $\langle\alpha\rangle^{\curvearrowright}\left\langle e_{\beta}: \beta<\mu\right\rangle$ where

$$
e_{\beta}=\left\{\begin{array}{l}
1 \text { if } \beta \in F \\
0 \text { if } \beta \notin F .
\end{array}\right.
$$

We claim that $f$ is an unbounded cofinal map. Let $X \subseteq G$ be unbounded. Then either (i) the set

$$
\left\{\alpha<\operatorname{cf} C:\left(T \uparrow c_{\alpha}\right) \backslash \bigcup_{\beta \in F}\left(T \uparrow s_{\beta}\right) \in X \text { for some } F \in[\mu]^{<\omega}\right\}
$$

is unbounded in $\operatorname{cf} C$, or (ii) the set

$$
\left\{\gamma<\mu:\left(T \uparrow c_{\alpha}\right) \backslash \bigcup_{\beta \in F}\left(T \uparrow s_{\beta}\right) \in X \text { for some } \alpha<\operatorname{cf} C, F \in[\mu]^{<\omega} \text { with } \gamma \in F\right\}
$$

is infinite. In case (i), the set of first coordinates of elements of $f[X]$ is unbounded in $\operatorname{cf} C$. In case (ii), there are infinitely many $\beta<\mu$ at which some element of $f[X]$ has the value 1 . In either case, $f[X]$ is unbounded in $\left(\operatorname{cf} C \times \prod_{\beta<\mu}^{\mathrm{w}}\{0,1\}, \leq\right)$.

Now suppose $X \subseteq G$ is a cofinal subset. Let $p=\left\langle\alpha, e_{0}, e_{1}, \ldots, e_{\beta}, \ldots\right\rangle \in(\operatorname{cf} C \times$ $\left.\prod_{\beta<\mu}^{\mathrm{w}}\{0,1\}, \leq\right)$. Set $F=\left\{\beta<\mu: e_{\beta}=1\right\}$. As $X$ is cofinal in $G$, there is some $x \in X$ such that $x \subseteq\left(T \uparrow c_{\alpha}\right) \backslash \bigcup_{\beta \in F}\left(T \uparrow s_{\beta}\right)$. Then $f(x) \geq p$. Thus $f[X]$ is cofinal in $\left(\operatorname{cf} C \times \prod_{\beta<\mu}^{\mathrm{w}}\{0,1\}, \leq\right)$.

Then since $G$ is closed under finite intersection and generates $\mathcal{U}$,

$$
(\mathcal{U}, \supseteq) \equiv_{T}(G, \supseteq) \equiv_{T}\left(\operatorname{cf} C \times \prod_{\beta<\mu}^{\mathrm{w}}\{0,1\}, \leq\right) .
$$

From Theorem 28 and Fact 26, we have the following corollary:

Corollary 29. Let $\kappa>\omega$ and let $T$ be a tree of size $\kappa$. Then Treealg $T$ has an ultrafilter with type $\left([\kappa]^{<\omega}, \subseteq\right)$ if and only if $T$ has an initial chain with $\kappa$-many immediate successors. 
From Theorem 28 we can describe the Tukey spectrum of a tree algebra.

Theorem 30. Let $T$ be a tree. The Tukey spectrum of Treealg $T$ consists of those Tukey types $\left(\operatorname{cf} C \times \prod_{\beta<\mu}^{\mathrm{w}}\{0,1\}, \leq\right)$ where $C \subseteq T$ is an initial chain with $\mu$-many immediate successors.

Finally, we turn our attention to the broader class of pseudo-tree algebras. Corollary 29 showed that the tree algebra on an uncountable tree $T$ has an ultrafilter of top Tukey type if and only if there is an initial chain $C$ in $T$ with $|\operatorname{imm}(C)|=|T|$. One possible pseudo-tree analog of "having an initial chain with $|T|$-many immediate successors" would be "having an initial chain $C$ with $\varepsilon_{C}=|T|$ ", and so one could ask whether the ultrafilter corresponding to such an initial chain has top Tukey type. The answer is "no". For example, let $T$ be a linear order of order type $\omega_{1}+1+\omega_{1}^{*}$ (this is also a pseudo-tree), and let $C$ consist of the first $\omega_{1}$-many elements of $T$. Then by Theorem 24, the ultrafilter corresponding to $C$ has type $\left(\omega_{1}, \leq\right)$, which is strictly less than the top type for Treealg $T$ by Fact 10 (4).

The next Fact shows that uncountable pseudo-tree algebras always have ultrafilters whose Tukey type is strictly below the maximum type.

Fact 31. Let $T$ be an uncountable pseudo-tree. Then there is an ultrafilter $\mathcal{U}$ on Treealg $T$ whose Tukey type is strictly less than that of $\left([|T|]^{<\omega}, \subseteq\right)$.

Proof. Let $T$ be an uncountable pseudo-tree. Let $C$ be any initial chain such that for no $t \in T$ is $t>C$, and let $\mathcal{U}$ be the ultrafilter corresponding to $C$. Then $C$ is generated by $G=\{T \uparrow c: c \in C\}$, so that $(U, \supseteq) \equiv_{T}(G, \supseteq) \equiv_{T}(\kappa, \leq)$ for some cardinal $\kappa$. Since $T$ is uncountable, $(\kappa, \leq) \not_{T}\left([|T|]^{<\omega}, \subseteq\right)$.

Proposition 32. Let $\lambda$ be any cardinal (finite or infinite) and let $\left\{\kappa_{\delta}: \delta<\lambda\right\}$ be a set of regular cardinals, each of which is either infinite or else 2 . Then there is a pseudo-tree $T$ and an ultrafilter $\mathcal{U}$ on Treealg $T$ such that $(\mathcal{U}, \supseteq) \equiv_{T} \prod_{\delta<\lambda}^{\mathrm{w}} \kappa_{\delta}$.

Proof. Let regular cardinals $\kappa_{\delta}$, for $\delta<\lambda$, be given, where each $\kappa_{\delta}$ is either infinite or else 2. For each $\delta<\lambda$, let $\kappa_{\delta}^{\prime}=\kappa_{\delta}$ if $\kappa_{\delta}$ is an infinite cardinal, and let $\kappa_{\delta}^{\prime}=1$ if $\kappa_{\delta}=2$. Let $T$ be the pseudo-tree constructed by putting above a single root $r$ $\lambda$-many pairwise-incomparable linear orders $T_{\delta}=\left\{t_{\alpha}^{\delta}: \alpha<\kappa_{\delta}^{\prime}\right\}$ where each $T_{\delta}$ is an inverted copy of $\kappa_{\delta}^{\prime}$ - that is, $T_{\delta}$ is isomorphic to $\left(\kappa_{\delta}^{\prime}\right)^{*}$. Let $A=$ Treealg $T$ be the pseudo-tree algebra on $T$. Let $C=\{r\}$ and let $\mathcal{U}$ be the ultrafilter associated with $C$. Then $\mathcal{U}=\langle G\rangle$ where

$$
G=\left\{(T \uparrow r) \backslash \bigcup_{s \in S}(T \uparrow s): S \text { is a finite antichain of elements above } r\right\} .
$$

Since any such antichain $S$ consists of at most one element $t_{\alpha_{\delta}}^{\delta}$ from each $T_{\delta}$, for $\delta<\lambda$, a typical element of $G$ is of the form $(T \uparrow r) \backslash \bigcup_{\delta \in F}\left(T \uparrow t_{\alpha_{\delta}}^{\delta}\right)$ for some $F \in[\lambda]^{<\omega}$ and $\alpha_{\delta}<\kappa_{\delta}^{\prime}, \delta \in F$. Also note that $(G, \supseteq) \equiv_{T}(\mathcal{U}, \supseteq)$, since $G$ is cofinal in $\mathcal{U}$.

Define $f:(G, \supseteq) \rightarrow\left(\prod_{\delta<\lambda}^{\mathrm{w}} \kappa_{\delta}, \leq\right)$ by

$$
f\left((T \uparrow r) \backslash \bigcup_{\delta \in F}\left(T \uparrow t_{\alpha_{\delta}}^{\delta}\right)\right)=\left\langle\beta_{\delta}: \delta<\lambda\right\rangle
$$

where, for $\delta<\lambda$,

$$
\beta_{\delta}= \begin{cases}0 & \text { if } \delta \notin F \\ 1+\alpha_{\delta} & \text { if } \delta \in F\end{cases}
$$


(That is: in those coordinates where no part of the chain $T_{\delta}$ has been excluded, let $\beta_{\delta}=0$; and in those coordinates where $T_{\delta}$ has been cut-off at $t_{\alpha_{\delta}}^{\delta}$, let $\beta_{\delta}=1+\alpha_{\delta}$.) Then $f$ is a bijection between $(G, \supseteq)$ and $\left(\prod_{\delta<\lambda}^{\mathrm{w}} \kappa_{\delta}, \leq\right)$ such that for all $g, g^{\prime} \in G$, $g \leq g^{\prime} \leftrightarrow f(g) \leq f\left(g^{\prime}\right)$. By Fact $8(2),(\mathcal{U}, \supseteq) \equiv_{T}(G, \supseteq) \equiv_{T}\left(\prod_{\delta<\lambda}^{\mathrm{w}} \kappa_{\delta}, \leq\right)$.

The following Lemma 34 distills the essential structure inside any set of approximate immediate successors above a chain in a pseudo-tree. This structure is given by the following notion of a $\lambda$-fan.

Definition 33. Let $C$ be an initial chain in a pseudo-tree $T$. A set $\Lambda=\left\{t_{\beta}^{\alpha}\right.$ : $\left.\alpha<\lambda, \beta<\theta_{\alpha}\right\}$ of elements of $T$ above $C$ is a $\lambda$-fan above $C$ if the following four conditions hold:

(1) $\Lambda$ is a set of approximate immediate successors of $C$.

(2) $\lambda$ is a cardinal and for each $\alpha<\lambda, \theta_{\alpha}$ is either equal to 1 or an infinite regular cardinal.

(3) For each $\alpha<\lambda,\left\{t_{\beta}^{\alpha}: \beta<\theta_{\alpha}\right\}$ is a strictly decreasing coinitial chain above $C$.

(4) For $\alpha<\alpha^{\prime}<\lambda, \beta<\theta_{\alpha}$, and $\beta^{\prime}<\theta_{\alpha^{\prime}}, t_{\beta}^{\alpha} \perp t_{\beta^{\prime}}^{\alpha^{\prime}}$.

Lemma 34. Let $T$ be a pseudo-tree and let $C$ be an initial chain in T. Given any set $S$ of approximate immediate successors of $C$, there is a $\lambda$-fan $\Lambda=\left\{t_{\beta}^{\alpha}: \alpha<\right.$ $\left.\lambda, \beta<\theta_{\alpha}\right\}$ above $C$ which is coinitial in $S$. Moreover, the cardinal $\lambda$ and set of cardinals $\left\{\theta_{\alpha}: \alpha<\lambda\right\}$ are uniquely determined by $C$.

Proof. Let $T$ be a pseudotree and let $C$ be an initial chain in $T$. Let $S \subseteq T$ be a set of approximate immediate successors of $C$ of minimal cardinality. Let $\kappa=|S|$, and enumerate the members of $S$ as $s_{\delta}$, for $\delta<\kappa$. For each $\delta<\kappa$, let $\gamma(\delta)$ be the least $\gamma<\kappa$ such that $\left(\left(T \downarrow s_{\gamma}\right) \backslash C\right) \cap\left(\left(T \downarrow s_{\delta}\right) \backslash C\right) \neq \emptyset$. Note that $\gamma(\delta) \leq \delta$. Observe that each $\left(T \downarrow s_{\delta}\right) \backslash C$ is "closed downwards above $C$ " - that is, if $t \in\left(T \downarrow s_{\delta}\right) \backslash C$ and $C<s \leq t$, then $s \in\left(T \downarrow s_{\delta}\right) \backslash C$.

Subclaim 1. $\left(\left(T \downarrow s_{\delta}\right) \backslash C\right) \cap\left(\left(T \downarrow s_{\eta}\right) \backslash C\right) \neq \emptyset$ if and only if $\gamma(\delta)=\gamma(\eta)$.

Proof. First suppose $\left(\left(T \downarrow s_{\delta}\right) \backslash C\right) \cap\left(\left(T \downarrow s_{\eta}\right) \backslash C\right) \neq \emptyset$. Since also $\left(\left(T \downarrow s_{\delta}\right) \backslash C\right) \cap$ $\left(\left(T \downarrow s_{\gamma(\delta)}\right) \backslash C\right) \neq \emptyset$ and all of these sets are downwards-closed linear orders above $C$, we have $\left(\left(T \downarrow s_{\eta}\right) \backslash C\right) \cap\left(\left(T \downarrow s_{\gamma(\delta)}\right) \backslash C\right) \neq \emptyset$. Then $\gamma(\eta) \leq \gamma(\delta)$ by minimality of $\gamma(\eta)$. Similarly $\gamma(\delta) \leq \gamma(\eta)$.

Now suppose that $\gamma(\delta)=\gamma(\eta)$. Then $\left(\left(T \downarrow s_{\delta}\right) \backslash C\right) \cap\left(\left(T \downarrow s_{\gamma(\delta)}\right) \backslash C\right) \neq \emptyset$ and $\left(\left(T \downarrow s_{\eta}\right) \backslash C\right) \cap\left(\left(T \downarrow s_{\gamma(\delta)}\right) \backslash C\right) \neq \emptyset$. It follows that $\left(\left(T \downarrow s_{\delta}\right) \backslash C\right) \cap\left(\left(T \downarrow s_{\eta}\right) \backslash C\right) \neq$ $\emptyset$.

By Subclaim 1, $\left(T \downarrow s_{\delta}\right) \backslash C$ and $\left(T \downarrow s_{\eta}\right) \backslash C$ are disjoint if and only if $\gamma(\delta) \neq$ $\gamma(\eta)$. Let $\lambda$ be the cardinality of the set $\{\gamma(\delta): \delta<\kappa\}$, and enumerate the set $\left\{s_{\gamma(\delta)}: \delta<\kappa\right\}$ as $\left\{t_{0}^{\alpha}: \alpha<\lambda\right\}$. For each $\alpha<\lambda$, let $\theta_{\alpha}$ be the coinitiality of $\left(T \downarrow t_{0}^{\alpha}\right) \backslash C$, and let $\left\{t_{\beta}^{\alpha}: \beta<\theta_{\alpha}\right\}$ be a decreasing coinitial sequence in $\left(T \downarrow t_{0}^{\alpha}\right) \backslash C$. It follows that (2) - (4) in the definition of a $\lambda$-fan hold.

Subclaim 2. Let $\Lambda=\left\{t_{\beta}^{\alpha}: \alpha<\lambda, \beta<\theta_{\alpha}\right\}$. Then $\Lambda$ is a set of approximate immediate successors of $C$.

Proof. First note that $C<\Lambda$. Suppose $r>C$. As $S$ is a set of approximate immediate successors of $C$, there is an $s_{\delta} \in S$ with $C<s_{\delta} \leq r$. We have ( $T \downarrow$ $\left.\left.s_{\delta}\right) \backslash C\right) \cap\left(\left(T \downarrow s_{\gamma(\delta)}\right) \backslash C\right) \neq \emptyset$. Say $s_{\gamma(\delta)}=t_{0}^{\delta}$ for some $\delta<\lambda$. Pick $\beta$ large enough so 
that $t_{\beta}^{\alpha} \in\left(\left(T \downarrow s_{\delta}\right) \backslash C\right) \cap\left(\left(T \downarrow s_{\gamma(\delta)}\right) \backslash C\right)$. Then $t_{\beta}^{\alpha} \in \Lambda$ and $C<t_{\beta}^{\alpha} \leq s_{\delta} \leq r$. Thus $\Lambda$ is a set of approximate immediate successors of $C$; hence, Subclaim 2 holds.

Thus, (1) in the definition of $\lambda$-fan holds, so $\Lambda$ is a $\lambda$-fan above $C$.

Now suppose $S^{\prime}$ is another set of approximate immediate successors of $C$ and that $\Lambda^{\prime}=\left\{u_{\zeta}^{\xi}: \xi<\lambda^{\prime}, \zeta<\theta_{\xi}^{\prime}\right\} \subseteq S^{\prime}$ is a $\lambda^{\prime}$-fan above $C$. For each $\alpha<\lambda$, let $S_{\alpha}$ denote $\left\{t_{\beta}^{\alpha}: \beta<\theta_{\alpha}\right\}$ and for each $\xi<\lambda^{\prime}$, let $S_{\xi}^{\prime}$ denote $\left\{u_{\zeta}^{\xi}: \zeta<\theta_{\xi}^{\prime}\right\}$. Let $\bar{S}_{\alpha}$ denote $\left\{t \in T: t>C\right.$ and $\left.\exists \beta<\theta_{\alpha}\left(t \leq t_{\beta}^{\alpha}\right)\right\}$, and $\bar{S}_{\xi}^{\prime}$ denote $\{t \in T: t>C$ and $\left.\exists \zeta<\theta_{\xi}^{\prime}\left(t \leq u_{\zeta}^{\xi}\right)\right\}$. For each $\alpha<\lambda$, there is a $\xi(\alpha)<\lambda^{\prime}$ and a $\zeta(\alpha)<\theta_{\xi(\alpha)}^{\prime}$ such that $u_{\zeta(\alpha)}^{\xi(\alpha)} \leq t_{0}^{\alpha}$. Thus, $\bar{S}_{\alpha}$ and $\bar{S}_{\xi(\alpha)}^{\prime}$ have a common coinitial segment above $C$. By property (4) of a $\lambda$-fan, for all $\xi^{\prime} \neq \xi(\alpha), \bar{S}_{\xi^{\prime}}^{\prime} \cap \bar{S}_{\xi(\alpha)}^{\prime}=\emptyset$. Therefore, this $\xi(\alpha)$ is unique. Define $\varphi(\alpha)$ to be this $\xi(\alpha)$. This defines a one-to-one function $\varphi: \lambda \rightarrow \lambda^{\prime}$. Since for each $\xi<\lambda^{\prime}$ there is an $\alpha(\xi)<\lambda$ and a $\beta(\xi)$ such that $t_{\beta(\xi)}^{\alpha(\xi)} \leq u_{0}^{\xi}$, a similar argument reveals that the function $\varphi$ is also onto $\lambda^{\prime}$. Thus, $\varphi$ is a bijection; hence $\lambda=\lambda^{\prime}$. As noted above, for each $\alpha<\lambda, \bar{S}_{\alpha}$ and $\bar{S}_{\varphi(\alpha)}^{\prime}$ have a common coinitial segment above $C$. The coinitiality of this segment must simultaneously be equal to $\theta_{\alpha}$ and $\theta_{\varphi(\alpha)}^{\prime}$. Hence, $\theta_{\varphi(\alpha)}^{\prime}=\theta_{\alpha}$. Therefore, the cardinals representing any $\lambda$-fan above $C$ are uniquely determined by $C$.

The previous lemma is now applied to characterize the Tukey types of ultrafilters on pseudo-tree algebras.

Theorem 35. Let $T$ be a pseudo-tree and let $\mathcal{U}$ be an ultrafilter on Treealg $T$. Then $\mathcal{U}$ is Tukey equivalent to $\left(\mu \times \prod_{\alpha<\lambda}^{\mathrm{w}} \kappa_{\alpha}, \leq\right)$, where $\mu$ is either 1 or an infinite regular cardinal, $\lambda$ is some cardinal, and for $\alpha<\lambda, \kappa_{\alpha}$ is either 2 or else an infinite regular cardinal.

Proof. Let $T$ be a pseudotree and let $\mathcal{U}$ an ultrafilter on Treealg $T$. Let $C=\phi(\mathcal{U})$ be the initial chain corresponding to $\mathcal{U}$ and let $S \subseteq T$ be a set of approximate immediate successors of $C$ of minimal cardinality. By Lemma 34, there is a coinitial subset $\Lambda \subseteq S$ which is a $\lambda$-fan above $C$ of the form $\left\{t_{\beta}^{\alpha}: \alpha<\lambda, \beta<\theta_{\alpha}\right\}$ which is coinitial in $S$, where $\lambda$ is a cardinal, each $\theta_{\alpha}$ is either 1 or an infinite regular cardinal, and these cardinals are uniquely determined by $C$. For each $\alpha<\lambda$, let $\kappa_{\alpha}=1+\theta_{\alpha}$. Shifting the lower indices of the members of $\Lambda$ by one and letting $s_{1+\beta}^{\alpha}=t_{\beta}^{\alpha}$, we re-write $\Lambda$ as $\left\{s_{\beta}^{\alpha}: \alpha<\lambda, 1 \leq \beta<\kappa_{\alpha}\right\}$.

Let $\mu=\operatorname{cf} C$, and let $\left\{c_{\xi}: \xi<\mu\right\}$ be an increasing cofinal sequence in $C$. Then $\mathcal{U}=\langle G\rangle$ where

$$
G=\left\{\left(T \uparrow c_{\xi}\right) \backslash \bigcup_{s \in F}(T \uparrow s): \xi<\mu \text { and } F \text { is a finite antichain in } \Lambda\right\},
$$

since $\Lambda$ is a set of approximate immediate successors of $C$. We claim that $(G, \supseteq) \equiv_{T}$ $\left(\mu \times \prod_{\alpha<\lambda}^{\mathrm{w}} \kappa_{\alpha}, \leq\right)$. Define a map $f: G \rightarrow \mu \times \prod_{\alpha<\lambda}^{\mathrm{w}} \kappa_{\alpha}$ by $f\left(\left(T \uparrow c_{\xi}\right) \backslash \bigcup_{s \in F}(T \uparrow\right.$ $s))=\langle\xi\rangle^{\frown}\left\langle\varepsilon_{\alpha}: \alpha<\lambda\right\rangle$, where for $\alpha<\lambda$,

$$
\varepsilon_{\alpha}=\left\{\begin{array}{l}
0, \text { if } s_{\beta}^{\alpha} \notin F \text { for all } 1 \leq \beta<\kappa_{\alpha} \\
\beta, \text { if } s_{\beta}^{\alpha} \in F \text { for some } 1 \leq \beta<\kappa_{\alpha} .
\end{array}\right.
$$

Note that $f$ is a bijection which preserves order both ways; that is, for $g, g^{\prime} \in G$, $g \leq g^{\prime} \leftrightarrow f(g) \leq f\left(g^{\prime}\right)$. Thus $(\mathcal{U}, \subseteq) \equiv_{T}(G, \supseteq) \equiv_{T} \mu \times \prod_{\alpha<\lambda}^{\mathrm{w}} \kappa_{\alpha}$, by Fact $8(2)$ 
Note that this is consistent with what we already know about ultrafilters on tree algebras from Corollary 29 , because for any $\kappa,\left([\kappa]^{<\omega}, \subseteq\right) \equiv_{T} \prod_{\alpha<\kappa}^{\mathrm{w}}\{0,1\}$.

Since by Lemma 34, every set of approximate immediate successors of an initial chain $C$ has, up to isomorphism, the same $\lambda$-fan, we shall speak of the $\lambda$-fan above $C$. The characterization of the Tukey spectra of pseudo-trees follows immediately from Lemma 34 and Theorem 35. Further, combined with Fact 10 (2), they tell us exactly when a pseudo-tree has an ultrafilter with maximum Tukey type.

Theorem 36. Let $T$ be a pseudo-tree with a single root. The Tukey spectrum of Treealg $T$ consists of exactly those types $\left(\operatorname{cf} C \times \prod_{\alpha<\lambda}^{\mathrm{w}} \kappa_{\alpha}, \leq\right)$, where $C$ is an initial chain with $\lambda$-fan of approximate immediate successors $\Lambda=\left\{s_{\beta}^{\alpha}: \alpha<\lambda, \beta<\theta_{\alpha}\right\}$ and $\kappa_{\alpha}=1+\theta_{\alpha}$.

If $|T|=\kappa$, then $T$ has an ultrafilter with maximal type $([\kappa]<\omega, \subseteq)$ if and only if there is an initial chain $C \subseteq T$ with a $\kappa$-fan $K=\left\{s_{\beta}^{\alpha}: \alpha<\kappa, \beta<\theta_{\alpha}\right\}$ of approximate immediate successors above $C$, where $\kappa$-many of the cardinals $\theta_{\alpha}$ are 1.

Remark. We point out that $\operatorname{cf} C \times \prod_{\alpha<\lambda}^{\mathrm{w}} \kappa_{\alpha}$ is really the same as $\prod_{\alpha<\lambda+1}^{\mathrm{w}} \kappa_{\alpha}$, where we define $\kappa_{\lambda}$ to be $\operatorname{cf} C$. We leave the $\operatorname{cf} C$ on the left to remind the reader of how the structure of the pseudo-tree influences the Tukey types of its ultrafilters.

\section{Questions}

We conclude with some open questions either motivating or arising from this paper.

Question 37. Characterize those Boolean algebras that have an ultrafilter of the maximum Tukey type, and characterize those Boolean algebras that have an ultrafilter of Tukey type strictly below the maximum.

Question 38. If $\mathbb{B}$ is an infinite Boolean algebra such that all ultrafilters on $\mathbb{B}$ have maximum Tukey type, is $\mathbb{B}$ necessarily a free algebra?

Or is the following possible?

Question 39. Does the completion of a free Boolean algebra have only ultrafilters which are of maximum Tukey type?

If not, can we at least rule out the remaining possible case of a cofinally scalene ultrafilter?

Question 40. Can the completion of a free Boolean algebra have an ultrafilter which is cofinally scalene; that is Tukey equivalent to $\prod_{\alpha<\lambda} \kappa_{\alpha}$, for some infinite $\lambda$ and each $\kappa_{\alpha} \geq \omega$ ? In particular, can the Cohen algebra have an ultrafilter Tukey equivalent to $\left(\omega^{\omega}, \leq\right)$ ?

Note that a positive answer to Question 40 would imply a negative answer to Question 39, and a positive answer to Question 39 would imply a negative answer to Question 38 .

Question 41. What are the Tukey spectra of superatomic Boolean algebras? 


\section{BiBLIOGRAPHY}

\section{REFERENCES}

[1] Andreas Blass, Natasha Dobrinen, and Dilip Raghavan. The next best thing to a p-point. page 35pp, 2013. Submitted.

[2] Jennifer Brown. Character of pseudo-tree algebras. Order, 2014. DOI 10.1007/s11083-0149338-4.

[3] Mahlon M. Day. Oriented systems. Duke Mathematical Journal, 11:201-229, 1944.

[4] Natasha Dobrinen. Survey on the Tukey theory of ultrafilters. Selected Topics in Combinatorial Analysis, Zbornik Radova, Mathematical Institutes of the Serbian Academy of Sciences, page $27 \mathrm{pp}$. To appear.

[5] Natasha Dobrinen. High dimensional Ellentuck spaces and initial chains in the Tukey structure of non-p-points. page 28pp, 2014. Submitted.

[6] Natasha Dobrinen, José Grigorio Mijares, and Timothy Trujillo. Topological Ramsey spaces from Fraïssé classes, Ramsey-classification theorems, and initial structures in the Tukey types of p-points. page 34pp. Submitted.

[7] Natasha Dobrinen and Stevo Todorcevic. A new class of Ramsey-classification Theorems and their applications in the Tukey theory of ultrafilters, Part 2. Transactions of the American Mathematical Society, page 34pp. To appear.

[8] Natasha Dobrinen and Stevo Todorcevic. Tukey types of ultrafilters. Illinois Journal of Mathematics, 55(3):907-951, 2011.

[9] Natasha Dobrinen and Stevo Todorcevic. A new class of Ramsey-classification Theorems and their applications in the Tukey theory of ultrafilters, Part 1. Transactions of the American Mathematical Society, 366(3):1659-1684, 2014.

[10] John Isbell. The category of cofinal types. II. Transactions of the American Mathematical Society, 116:394-416, 1965.

[11] S. Koppelberg and J.D. Monk. Pseudo-trees and Boolean algebras. Order, 8:359-374, 1992.

[12] Sabine Koppelberg. In J. Donald Monk and Robert Bonnet, editors, Handbook of Boolean Algebras, Vol. 1. North-Holland, 1989.

[13] David Milovich. Tukey classes of ultrafilters on $\omega$. Topology Proceedings, 32:351-362, 2008.

[14] David Milovich. Forbidden rectangles in compacta. Topology and its Applications, 159:31803189, 2012.

[15] Dilip Raghavan and Stevo Todorčević. Cofinal types of ultrafilters. Annals of Pure and Applied Logic, 163(3):185-199, 2012.

[16] Judy Roitman. Superatomic Boolean algebras. In J. Donald Monk and Robert Bonnet, editors, Handbook of Boolean Algebras, Vol. 3, pages 719-740. North-Holland, 1989.

[17] Jürgen Schmidt. Konfinalität. Zeitschrift für Mathematische Logik und Grundlagen der Mathematik, 1:271-303, 1955.

[18] Saharon Shelah. Proper and Improper Forcing. Springer-Verlag, second edition, 1998.

[19] Slawomir Solecki and Stevo Todorčević. Cofinal types of topological directed orders. Annales de L'Institut Fourier, 54(6):1877-1911, 2004.

[20] Stevo Todorčević. Directed sets and cofinal types. Transactions of the American Mathematical Society, 290(2):711-723, 1985.

[21] Stevo Todorčević. A classification of transitive relations on $\omega_{1}$. Proceedings of the London Mathematical Society, (3) 73(3):501-533, 1996.

[22] John W. Tukey. Convergence and uniformity in topology. Princeton University Press, 1940. E-mail address: jennifer.brown@csuci.edu

Department of Mathematics, California State University - Channel Islands, One University Drive, Camarillo, CA 93012 U.S.A.

E-mail address: natasha.dobrinen@du.edu

$U R L:$ http://web.cs.du.edu/ ndobrine

Department of Mathematics, University of Denver, 2280 S Vine St, Denver, CO 80208 U.S.A. 